\title{
CFD Analysis of Thermal Distribution in the Industrial Fermenter during Sterilization Process
}

\section{Pirooz AP ${ }^{1 *}$, Ahmad HS ${ }^{2}$ and Gholam RR ${ }^{1}$}

${ }^{1}$ Islamic Azad University, North Tehran Branch, Tehran, Iran

${ }^{2}$ Caspian Faculty of Engineering, College of Engineering, University of Tehran, Rezvanshar, Guilan, Iran

\begin{abstract}
In biological reactors, isolation of unwanted microorganisms by using sterilization is essential. One of the important reasons of microbial contamination in industrial fermenter is bioreactor poor design which causes to create dead spot during sterilization. Therefore, identifying the dead spots in the sterilization process is crucial which could be accomplished by computational fluid dynamics (CFD). In this study, for identifying the spots which their thermal distributions are not uniform (due to fermenter geometry deficiency), the sterilization process in a $140 \mathrm{~m} 3$ fermenter (without culture media) were modeled $\left(129-133^{\circ} \mathrm{C}\right.$ and $\left.15 \mathrm{~min}\right)$. Study of temperature profile, velocity and stream lines shows that in the manhole, around the coils, steam inputs and below the impeller on the agitator shaft are the places that the risk of dead spot creation is high.
\end{abstract}

Keywords: Penicillin G; Fermenter sterilization; Thermal distribution; Computation fluid dynamics

Abbreviations: CFD: Computation Fluid Dynamics; DCS: Distributed Control System; T: Time (s); No: The first concentration of spores $\left(\mathrm{mol} / \mathrm{m}^{3}\right) ; \mathrm{N}_{\mathrm{f}}$ : The final concentration of spores $\left(\mathrm{mol} / \mathrm{m}^{3}\right) ; \nabla$ : Delfactor; T: Temperature (0K); k: Destruction of micro-organisms factor; V: Velocity (m/s); P: Pressure (Pa); g: Acceleration due to gravity (ms $\left.{ }^{2}\right)$; $\rho$ : Density $\left(\mathrm{kg} / \mathrm{m}^{3}\right)$; E: Activation Energy $(\mathrm{KJ} / \mathrm{mol})$; $\mathrm{C}_{\mathrm{p}}$ : Specific heat capacity at pressure constant (J/kg.K).

\section{Introduction}

Sterilization process is one of the most important operations in food, pharmaceutical and medical industries. First step in the successful fermentation achievement is good sterilization. In most industries for increasing the efficiency and reliability, use wet steam. Good achievement sterilization depends on fermenter geometry, intended temperature, sterilization pressure and time. In most pharmaceutical companies using of biological reactors for fermentation process is very popular. In these kind of biological reactors, growth of specific microorganisms is desirable, so medium must be free of any unwanted microorganisms. Particular temperatures are proposed for assurance of microorganisms' inactivation which most common are $121^{\circ} \mathrm{C}$ for 30 minutes and $132^{\circ} \mathrm{C}$ for 4 minutes. Thermal influx depends on sterilization medium state (solid-liquid-gas) and physical and chemical properties of sterilization culture media. Existence of dead spots in the mixing systems caused by installed baffles and coils maybe have influence on a good sterilization. Because scaling up of agitating systems is difficult, so to study of thermal distribution in biological reactors, simulation tools i.e., CFD, are useful [1]. In recent years, application of CFD in many industries is in progress with higher rate. With the use of dominant mathematical equation and numerical solutions, fluid Behavior could be simulated from the perspective of mass, heat and momentum transfer [2,3]. Analysis of thermal distribution of canned milk sterilization by CFD was studied by the Dhayalin [4]. Sterilization by use of a CFD model in a canned food containing solid-liquid mixture was studied by the Farid and Ghani in 1999 [5]. By the use of CFD, pasteurization of milk was studied at 74$85^{\circ} \mathrm{C}$ by Anandpaul [6]. Varma and Kannan studied the sterilization process in various cans geometry. Also they simulated the natural heat transfer in cylindrical and coned shape cans by the use of CFD [7]. In 2012, Tang simulated the central temperature of orange juice in HTST sterilization model with the use of CFD [8]. In 1996, Datta studied the temperature and velocity profile in a cylindrical can of water with natural convection [9]. Sterilization of a viscose food in a cylindrical can in the $121^{\circ} \mathrm{C}$ of wall in the all dimensions was studied. Simultaneously solving of the momentum, energy and continuity equations resulted the temperature, velocity and stream line profile. Also Kumar simulated the temperature profile whereas the bottom and top of the can was isolated [10]. Modeling the continuous sterilization for killing the food contamination shows that simulation that accomplished with CFD can impressively help to modify food sterilization.

\section{Microorganisms destruction kinetic}

Microorganism's destruction kinetics could be considered as a first order reaction.

$\ln \frac{N_{t}}{N_{0}}=-k t$

In the destruction equation, $\mathrm{k}$ is similar with chemical equation constant in first order reaction. This constant is related to temperature and is expressed by Arrhenius equation.

$\ln \frac{N_{0}}{N_{t}}=k_{0} t e^{\frac{-E}{R T}}$

Del-factor is the fractional reduction in microorganism's population.

$\nabla=\ln \frac{N_{0}}{N_{t}}$

During the sterilization process, del-factor is the algebraic summation of heating, retention and cooling [11].

For optimization achievement in sterilization process, reducing the sterilization time in holding area is necessary. While the input pressure is constant, the optimum sterilization time is the least time needed for microorganism's complete destruction.

*Corresponding author: Pirooz AP, Islamic Azad University, North Tehran Branch, Tehran, Iran, Tel: +98263335212; E-mail: pirooz.asadi@yahoo.com

Received August 20, 2017; Accepted October 11, 2017; Published October 13 , 2017

Citation: Pirooz AP, Ahmad HS, Gholam RR (2017) CFD Analysis of Thermal Distribution in the Industrial Fermenter during Sterilization Process. J Bioprocess Biotech 7: 309. doi:10.4172/2155-9821.1000309

Copyright: (c) 2017 Pirooz AP, et al. This is an open-access article distributed unde the terms of the Creative Commons Attribution License, which permits unrestricted use, distribution, and reproduction in any medium, provided the original author and source are credited. 


\section{Experimental}

Experimental values of temperature were obtained from 3 temperature sensors to compare time needed to reach the desire temperature between experimental and modeling method. Fermenter we sterilized is belonging to Shifa Pharmed Company which produces Penicillin through fermentation process. In this study we used 3 temperature sensors. One of them was a temperature probe (JUMO RTD) and two others was temperature electrodes (pt-100) were mounted in different places. The first and main sensor was mounted in $5.2 \mathrm{~m}$ height of fermenter. Second sensor was mounted in sampling valve port about $2 \mathrm{~m}$ height of fermenter and the third sensor was mounted in $11 \mathrm{~m}$ height of fermenter. It should be noted that all of these sensors before installation were calibrated. Because sterilization process in industrial scale is costly, so testing was done twice. Sensors installation places are shown below. Sterilization was carried out by opening 3 steam valves and one exhaust valve. At first, the fermenter temperature was increased from $\mathrm{N}_{0}$ to $\mathrm{N}_{1}$. According to the Figures 1 and 2, heating time to reach $\mathrm{N}_{0}$ to $\mathrm{N}_{1}$ point is $\mathrm{t}_{1}$. After that heat flux must be kept constant to stay in holding area. The time needed to reach to the $\mathrm{N}_{2}$ is $\mathrm{t}_{2}$. Modeling is done between $\mathrm{N}_{1}$ and $\mathrm{N}_{2}$. After that cooling process is must be done. During the sterilization, fermenter is empty and mixer is off. Temperature was recorded regularly every $5 \mathrm{~min}$. For control of temperature and pressure, DCS controlling system was used which is under license of Siemens Company. In Figure 3, DSC controlling system environment has been shown.

\section{Computational fluid dynamics}

Dominant equations: With numerical method, Navier-Stokes and other dominant equations (Conservation of mass, energy and ...) can be solved by specific boundary conditions and get the different results etc., velocity changes, temperature and .... Navier-Stokes equations state Conservation of mass (Continuity equation), momentum (Newton's second law) and energy (First law of thermodynamics) [12].

1. Conservation of mass equation

$$
\frac{\partial \rho}{\partial t}+\nabla \cdot(\rho \overrightarrow{\mathrm{V}})=0
$$

2. Momentum equation

$$
\rho\left[\frac{\partial \vec{V}}{\partial t}+(\vec{V} \cdot \nabla) \vec{V}\right]=-\nabla P+\nabla \cdot(\eta \cdot \nabla \vec{V})+\rho g
$$

3. Energy equation

$$
\frac{\partial\left(\rho C_{P} T\right)}{\partial t}+\nabla \cdot\left(\rho \vec{V} C_{P} T\right)=\frac{\partial p}{\partial t}+\nabla \cdot(K \nabla T)
$$

Sterilization process modeling is used to reduce food contamination risk which proves that simulation for food sterilization carried out by CFD can be so useful [12-16]. In pharmaceutical industries to ferment specific kind of microorganisms that is named Penicillium chrysogenum which is used for many antibiotic productions, first step is microorganism's growth medium sterilization. Growth medium of this kind of biotechnological process is in big fermenters that their capacity is more than $120 \mathrm{~m}^{3}$ that must be free of unwanted and harmful bacteria. One of the most important parameters in industrial fermenters design is destroying the probable sterile dead points through the fermenter. Since the cost of production process and construction of these fermenters are high, so use of CFD for design, reducing the contamination risk and also sterilization process optimization is very important. As regards of scaling up of the mechanical mixing systems always is along with great fault, so in this project, dry sterilization of a $140 \mathrm{~m}^{3}$ fermenter was simulated and the gained results was compared and evaluated against experimental data.

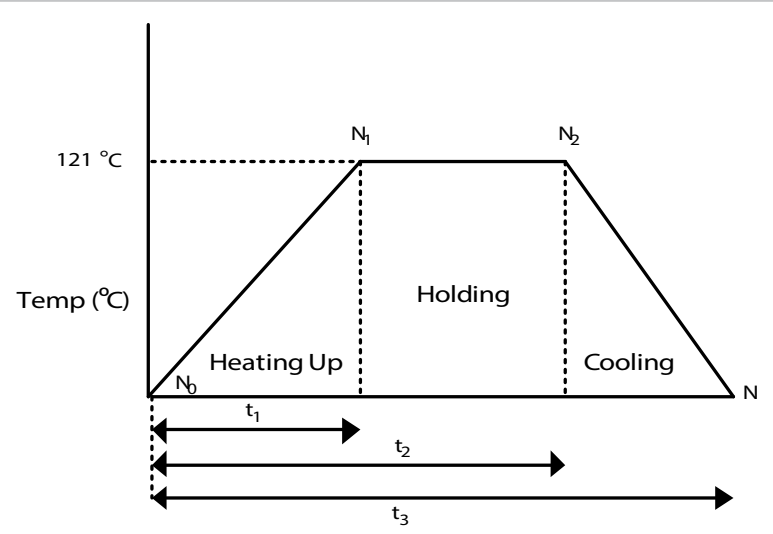

Figure 1: Temperature-time profile in a batch sterile.
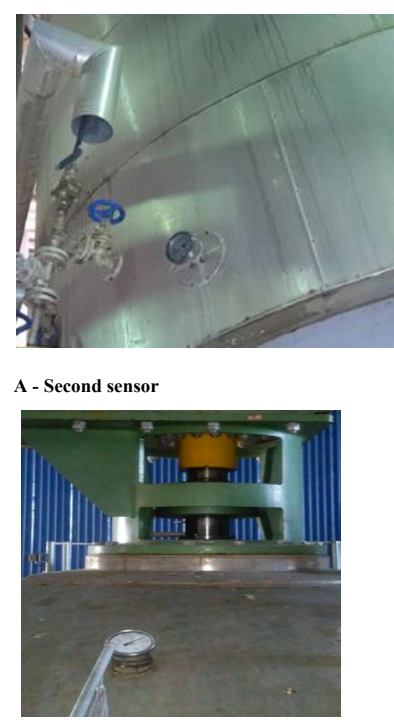

C - Third sensor

Figure 2: Various places of sensors.

Fermenter geometry: Geometry of sterilization process simulation is done in Gambit program. In this study volume of the fermenter are $140 \mathrm{~m}^{3}$ and the fermenter have 6 lines of coils which are also a kind of baffle. In geometry design which is made by Gambit, steam is injected to fermenter in 3 places and exhausted from top of the fermenter which is same as industrial process. In this study, simulation is analyzed in twodimensional. The mesh has been created in this study was unstructured quad grid with interval length of $10 \mathrm{~mm}$.

Boundary conditions: Due to the problem physics, boundary conditions are Stream and thermal properties determinative in boundaries. In this study, 3 kinds of boundary conditions; wall, pressure outlet and pressure inlet has been used. In this problem, from 3 points of fermenter where is pressure inlet, steam enters to fermenter with pressure of $0.17 \mathrm{MPa}$ gauge. In pressure outlet boundary condition, static pressure relatively exists from external exhaust. In viscous fluids by the default, is assumed that fluid movement on wall is non-slip. In this study fermenter wall and coil is consider as wall and because vessel is isolated, so heat exchange with outside is assumed to be zero. In this study Density Based solver is used which is designed for high velocity compressible streams. And Wet Steam was selected as simulation model. 
Citation: Pirooz AP, Ahmad HS, Gholam RR (2017) CFD Analysis of Thermal Distribution in the Industrial Fermenter during Sterilization Process. J Bioprocess Biotech 7: 309. doi:10.4172/2155-9821.1000309

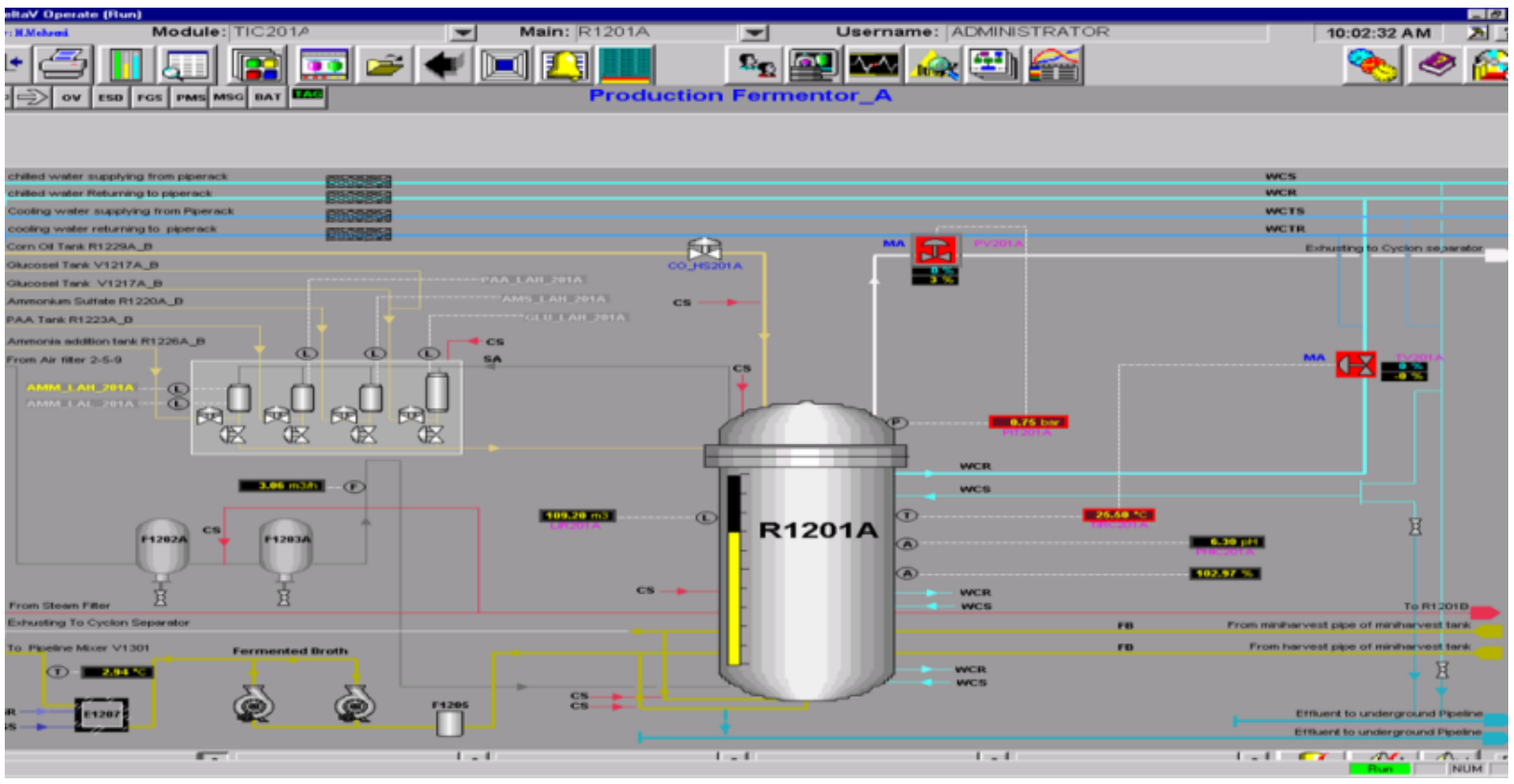

Figure 3: Fermenter controlling environment by use of DELTA $\vee$ software.

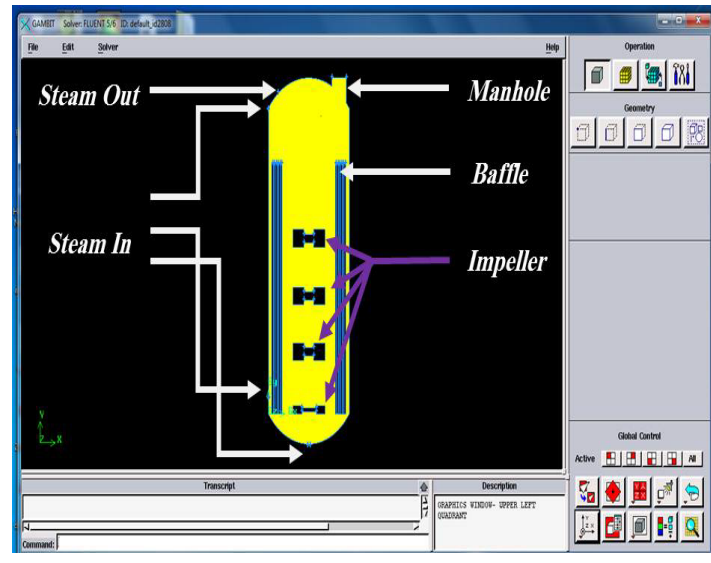

A - Gambit environment

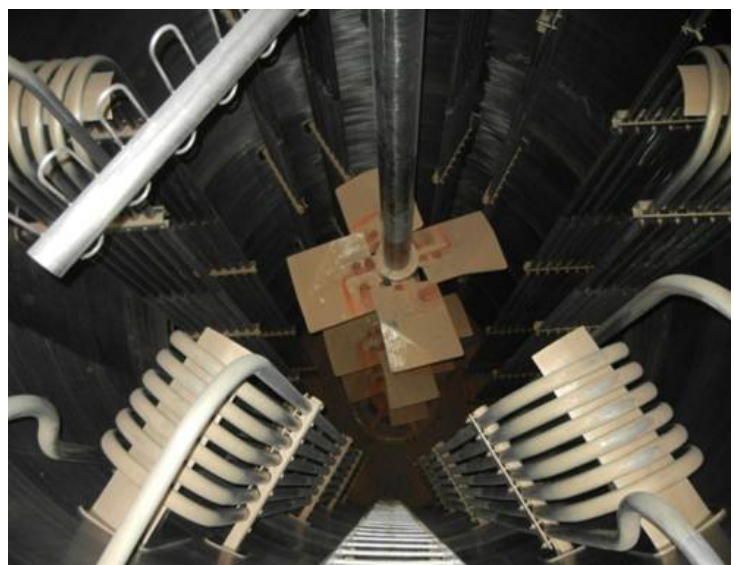

B - Industrial fermenter

Figure 4: Geometry of fermenter.

\section{Results and Discussion}

\section{Mesh analysis}

In this study two kinds of mesh structure have been used; Triangular and Quadrilateral. Obtained results show that Quadrilateral mesh provides more acceptable results than the Triangular one. Simulation case study was solved in two time intervals 0.1 and 0.01 seconds for study of time interval changes. As results show the measured errors will increase when time interval length increased, on the other hand using too short time intervals caused increasing the time of runs. So with the consideration of these reasons and measured error which is acceptable, time interval of 0.1 second was considered for all tests.

\section{Model study}

Temperature distribution: In Figures 4 and 5, temperature counters is shown. As see, in the state of holding time, temperature was constant among $129-130^{\circ} \mathrm{C}$. In the holding state, most points of fermenter have temperature among $129-130^{\circ} \mathrm{C}$, but the temperature distribution in these points was different. In manhole area, which was partly far from stream pattern path, temperature is relatively lower than other points. Top of the vessel is deviated from state of bent and somewhat increases the risk of dead points. Also in the shaft zone, due to mixers impeller which have role of barrier, temperature is lower than other points. Temperature profile around the impellers due to friction is more than other points. In Figure 6, temperature distribution around the impellers has been shown. According to the achieved temperature distribution, the risk of sterilization failure due to lack of dead points does not exist. Pressure drop created in the fermenter exhaust leads to increasing the temperature which the maximum temperature achieved is $133^{\circ} \mathrm{C}$. Temperature in the inlet port is $130^{\circ} \mathrm{C}$ and whatever getting away from inlet, temperature decreases. Left side and the right side of inlet stream along, temperature differences about 1 degree was observed 
Citation: Pirooz AP, Ahmad HS, Gholam RR (2017) CFD Analysis of Thermal Distribution in the Industrial Fermenter during Sterilization Process. J Bioprocess Biotech 7: 309. doi:10.4172/2155-9821.1000309

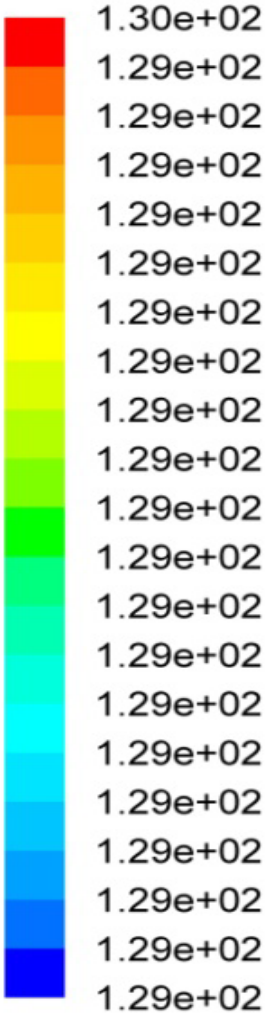

Figure 5: Temperature distribution in fermenter $\left({ }^{\circ} \mathrm{C}\right)$.

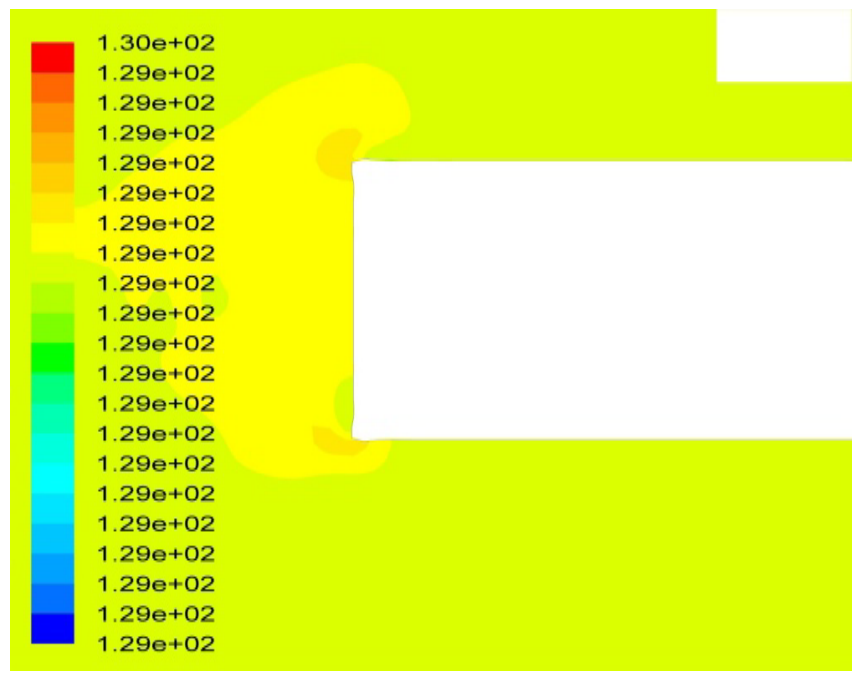

Figure 6: Temperature profile around the a part of impeller (C).

which its temperature is lower than inlet stream along temperature. In Figure 7, temperature counter of inlet is illustrated.

Pressure distribution: In Figure 8, pressure counter is showed. Pressure is variable among 0.144 to $0.17 \mathrm{MPa}$. As see, steam pressure of fermenter have a descending trend from bottom of vessel to top which in different distances decreases layer by layer in axial form. The maximum pressure observed was in exhaust and the minimum pressure observed was in the steam inlet port, which were 0.144 and $0.17 \mathrm{MPa}$ respectively.
As regards the fluid movement path was along the fermenter shaft, radial pressure drop was poor.

Velocity study: In Figure 9, counter of velocity is illustrated. For velocity formation, fluid movement along against the created tension along play important role. Velocity varies along the fluid stream in range of 0.17 to $150.2 \mathrm{~m} / \mathrm{s}$. due to pressure differences in inlet and outlet, velocity get maximum value of $150 \mathrm{~m} / \mathrm{s}$ in outlet. Due to crated velocity profile in wide range zones aside from shaft and manhole parts, velocity distribution of fluid in through the fermenter was nearly constant. In zones around the propeller blades, fluid ingression and egression had high velocity gradient. Velocity counters in low range is illustrated in below. In space between two propellers, radial variations of velocity along the stream are more than axial variations of that. In space between two propellers, axial variations are almost poor but in zones near the shaft and propeller blades end, axial distribution of fluid velocity is almost impressive. In the space above the first propeller, due to low shear stress, fluid velocity changes along the stream is inconsiderable.

Validation: As specified in diagram 2, time needed for industrial sterilization for reaching to the holding time is 60 minutes. CFD results represent it experimental temperature and simulation temperature at holding time zone differ 3 units which is negligible (Figures 10-12). Therefore, average results by the CFD show that the thermal distribution in fermenter is uniform except for small segments which justify the sterilization process success.

\section{Conclusion}

By the looking at the simulation results, we can assume temperature variation between 129 and $133^{\circ} \mathrm{C}$ as uniform thermal distribution. CFD results of temperature profile, velocity and stream lines shows that nearly all segments of fermenter have uniform temperature distribution. But in the manhole, around the coils, steam inputs and below the impeller on the agitator shaft are the places that the risk of dead spot creation

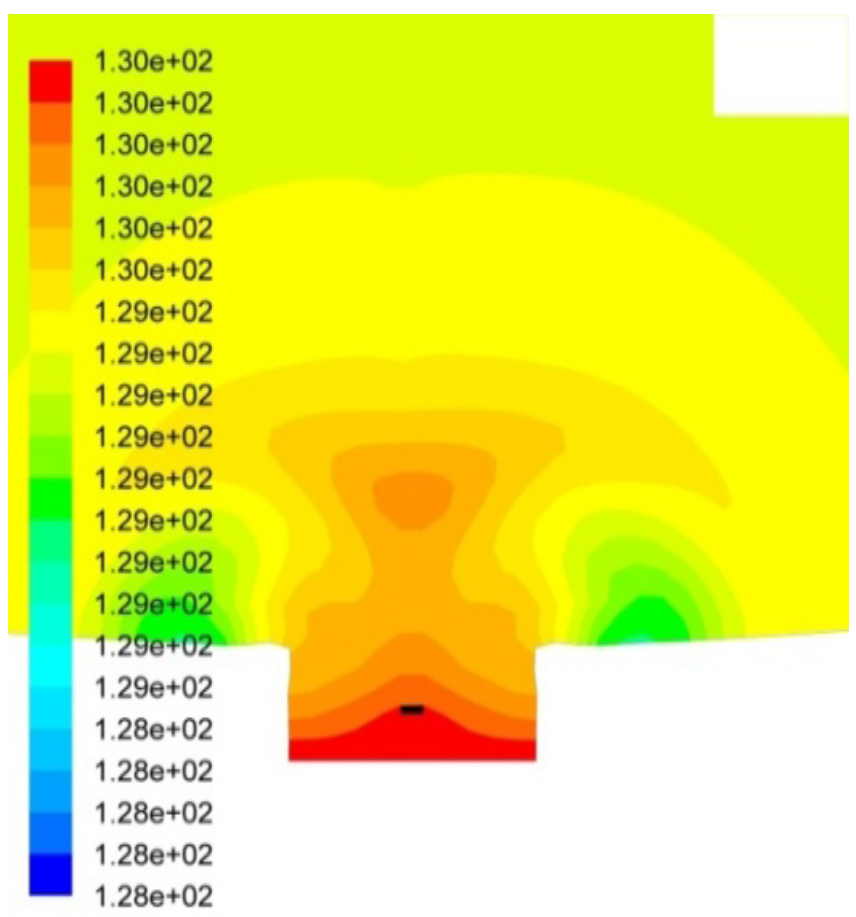

Figure 7: Temperature profile around the steam inlet $\left({ }^{\circ} \mathrm{C}\right)$. 


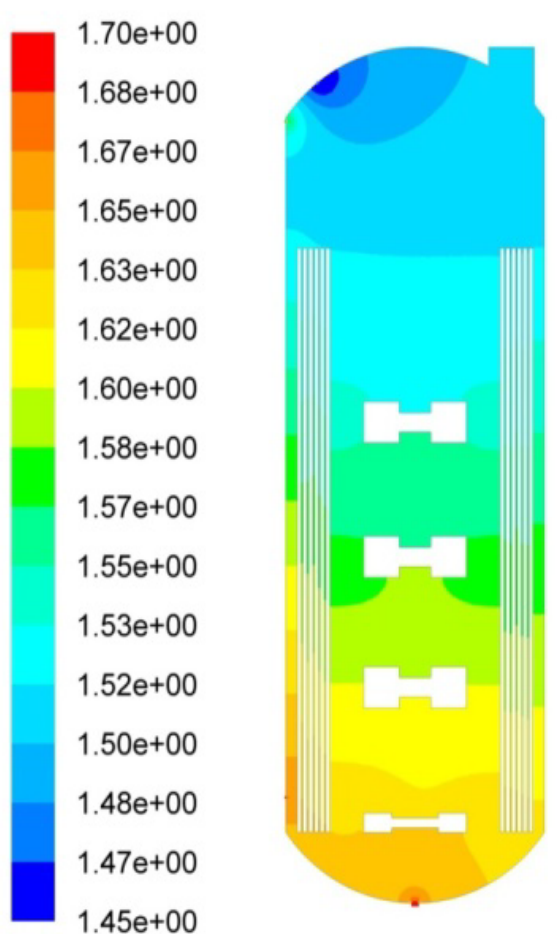

Figure 8: Pressure profile in fermenter (bar).

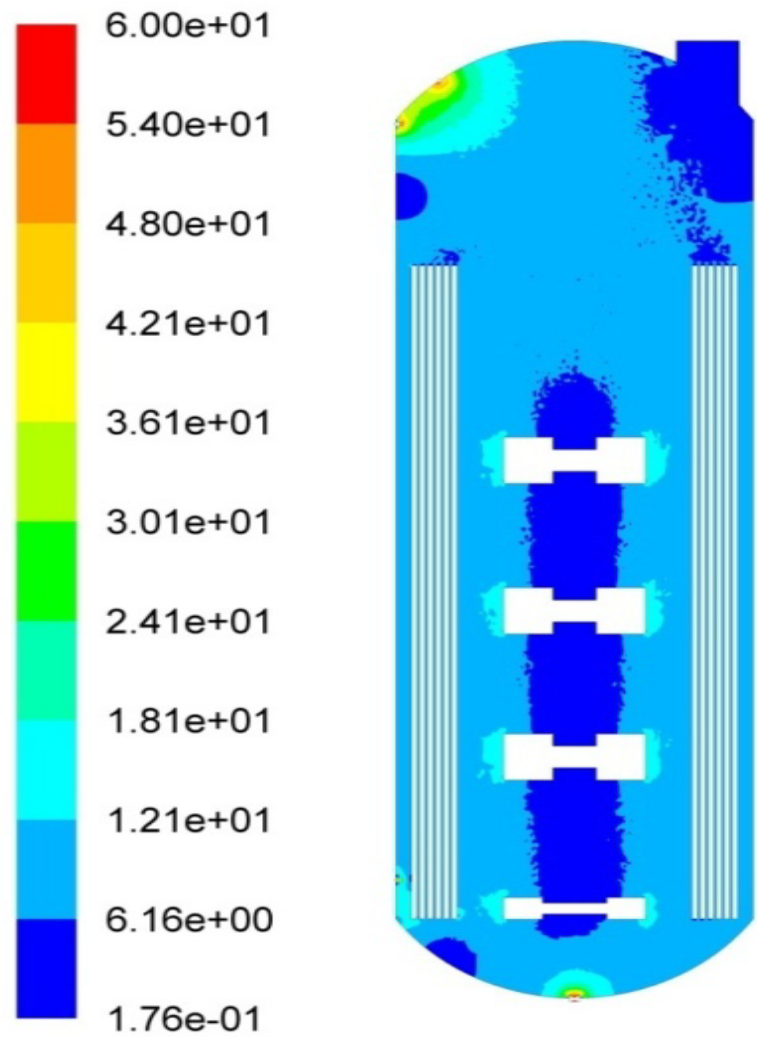

Figure 9: Velocity profile in fermenter $(10-60 \mathrm{~m} / \mathrm{s})$.
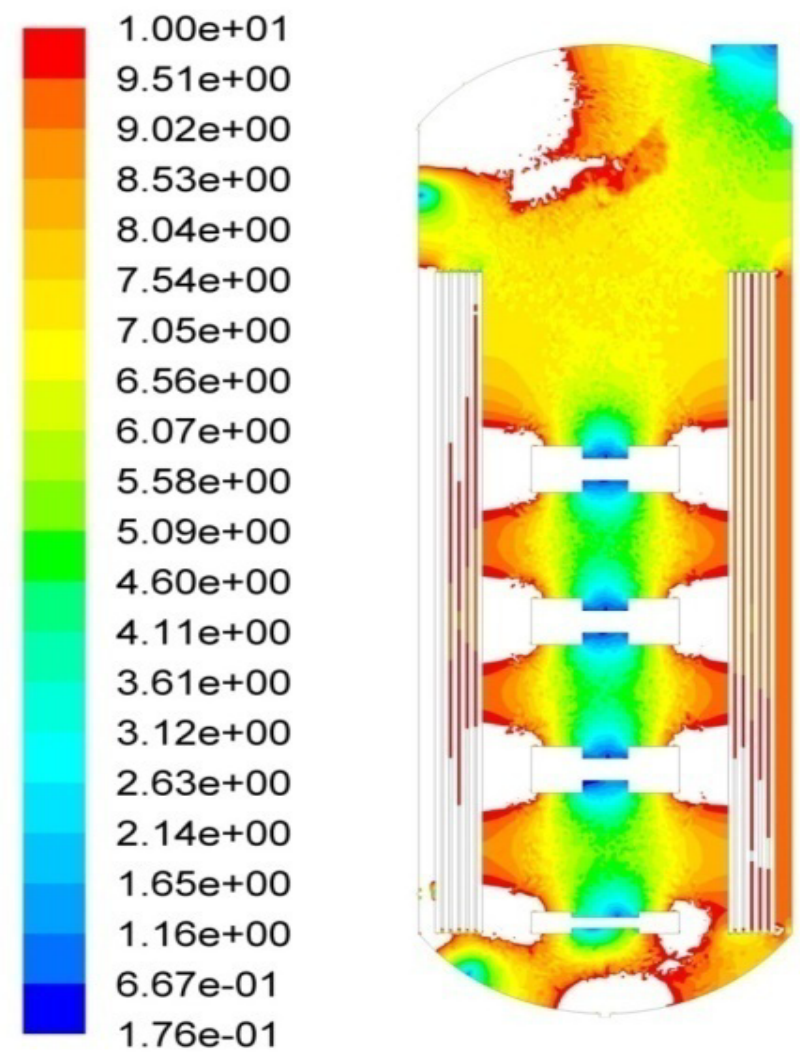

Figure 10: Velocity profile in fermenter (up to $10 \mathrm{~m} / \mathrm{s}$ ).

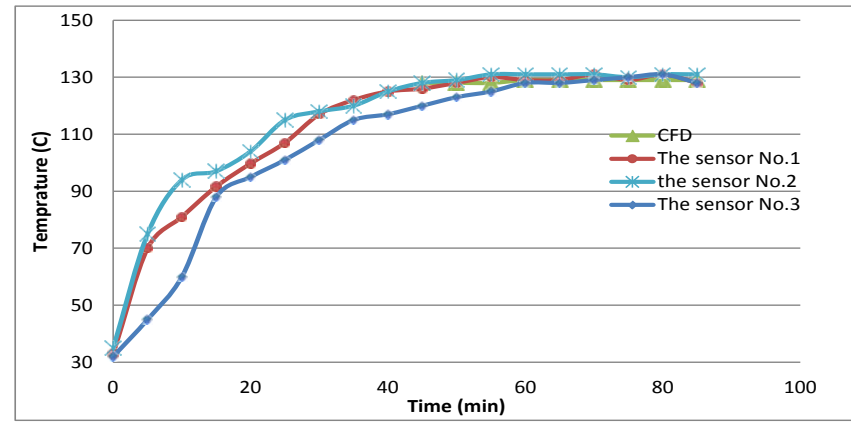

Figure 11: Analogy of experimental and simulation result during sterilization.

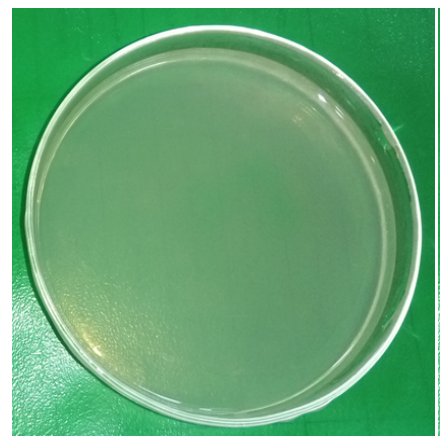

Plate number 2

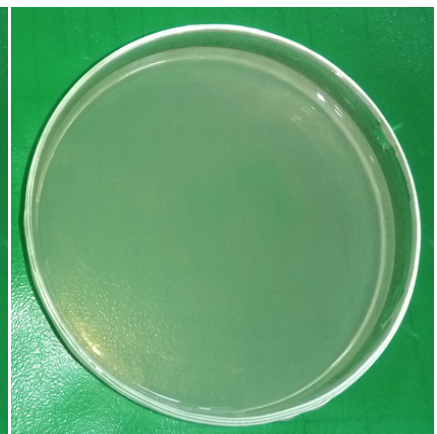

Plate number 1
Figure 12: Sampling from sterilized fermenter exhausted air. 
Citation: Pirooz AP, Ahmad HS, Gholam RR (2017) CFD Analysis of Thermal Distribution in the Industrial Fermenter during Sterilization Process. J Bioprocess Biotech 7: 309. doi:10.4172/2155-9821.1000309

Page 6 of 6

is high. Spots where steam velocity is low are dangerous places from the perspective of sterilization, because when contamination attached to the surface of the fermenters elements, could be removed only by a powerful force.

\section{Acknowledgements}

Authors wish to thank manager of Shifa Pharmed Company.

\section{References}

1. Xia B, Sun DW (2002) Applications of computational fluid dynamics (CFD) in the food industry: a review. Computers and Electronics in Agriculture 34: 5-24.

2. Abdul G, Farid MM, Chen XD (1999) Numerical simulation of natural convection heating of canned food by computational fluid dynamics. J Food Eng 41: 55-64.

3. Cordioli M, Rinaldi M, Copelli G, Casoli P, Barbanti D, et al. (2015) Computationa fluid dynamics (CFD) modelling and experimental validation of therma processing of canned fruit salad in glass jar. Journal of Food Engineering 150: 8

4. Dhayal P, Chhanwal N, Anandharamakrishnan C (2013) Heat Transfer Analysis of Sterilization of Canned Milk Using Computational Fluid Dynamics Simulations. Journal of Food Science and Engineering 3: 13-20.

5. Abdul AG, Farid MM, Chen XD (1999) An investigation of deactivation of bacteria in a canned liquid food during sterilization using computational fluid dynamics (CFD). J Food Eng 42: 207-214

6. Anandpaul D, Anishaparvin A, Anandharamakrishnan C (2011) Computational fluid dynamics studies on pasteurization of canned milk. Int J Dairy Technol 64: 305-313.

7. Mayank AK (2005) Enhanced food sterilization through inclination of the container walls and geometry modifications. Int J Heat Mass Transfer 48: 37533762.

8. Tang Y, Xie J, Wang J, Zhang Z, Gu C, et al. (2012) Theoretical Study and 3D CFD Simulation of Temperature Distribution through HTST for Orange Juice. Communications in Computer and Information Science 326: 1-7.

9. Datta AK, Teixeira AA (1988) Numerically Predicted Transient Temperature and Velocity Profiles during Natural Convection Heating of Canned Liquid Foods. Journal of Food Science 53: 191-195.

10. Kumar A, Bhattacharya M, Blaylock J (1990) Numerical simulation of natural convection heating of canned thick viscous liquid food products. J Food Sci 55: 9-15.

11. Thamer T, Mohammed J, Salah FT, Obaidi NF (2009) Study of the Optimization Condition of Batch Sterilization Using Genetic Algorithm. Eng \& Tech Journal 27: $1-5$.

12. Tattiyakul J, Rao MA, Datta AK (2001) Simulation of heat transfer to a canned corn starch dispersion subjected to axial rotation. Chemical Engineering and Processing 40: 391-399.

13. Jung A, Fryer PJ (1999) Quality Optimization of safe foods: Computational modelling of a continuous sterilization process. Chem Eng Sci 54: 14.

14. Kannan A, Gowri Sankar PS (2008) Heat analysis of canned food sterilization in a still retort. J Food Eng 88: 16-20.

15. Abdul G, Farid M (2004) A new computational technique for the estimation of sterilization time in canned food. Chem Eng Proc 43: 157-167.

16. Mahesh N, Kannan A (2006) CFD studies on natural convective heating of canned food in conical and cylindrical containers. J Food Eng 77: 1024-1036. 